\title{
La investigación en la UNAM: su uso y difusión
}

\author{
Rosa María Fernández de Zamora ${ }^{1}$
}

\section{RESUMEN}

Se con si de ra la im por tan cia que tie nen las la bo res de in ves ti ga ción en la UNAM tan to en el cam po cien tífi co- téc ni co como en el de huma ni da des y cien cias so ciales. Se hace una re fe ren cia es pe cial a la in ves ti ga ción bibliotecoló gi ca. Seex ponen las modalida des de difur sión de la in ves ti ga ción ha cien do re co men da cio nes para me jo rar los ca na les de di fusión que se tie nen ac tual men te, de tal ma nea que la investigación producida en la UNAM sea conocida con oportunidad tanto dentro como fuera de ella.

\section{ABSTRACT}

The im por tan ce of re search ac ti vi ties in the UNAM, bouth in the scien ti fic-te chni cal field as web as in the hu ma ni ties and so cial sciences, is considered. Special men tion is made of li brary re search. The means used to pu blish re search are discussed. Recommendations are also made to im prove the channels of commu ni ca tion cu rrently used so that re search produced in the UNAM can beco me known both within the university and outside as early as possible.

In ves ti gar vie ne del la tíninvestigare que sig nifica ha cer di ligencias para des cu brir algo o en con trar so lu ción a un pro ble ma. Significa, pues, búsqueda, indagación, averiguación, pesquisa.

La investigación surge cuando se tiene conciencia o necesidad de resolver un problema. Las acciones realizadas para alcanzar esa solución constituyen la investigación propiamente dicha.

La in ves ti ga ción, por tan to, "es aquel la ac ti vi dad, teó ri ca o prác tica, cien tífi ca o hu ma nís ti ca, aso cia da a la bús que da de nue vos conocimientos. La investigación nos permite conocer cada vez me jor el mun do que nos ro dea y ha de sem pe ña do un pa pel pri mordial en el de sa rro llo del hom bre en to dos sus ám bi tos". (Asti Vera)

El hom bre siem pre está en bus ca de nue vos co no ci mien tos que le permitan sa tis fa cersu am pliagama de ne ce si da des, por ello la finalidad última de la investigación es el saber. Aristóteles decía que apren der es el más gran de de los pla ce res no so la men te para el fi ló so fo sino tam bién para el res to de la hu ma ni dad por pe queña que sea su capacidad para ello.

La in ves ti ga ción pre sen ta una dua li dad: la in ves ti ga ción ociencia pura y la investigación o ciencia aplicada, mismas que podrían describirse con las palabras conocimiento y acción. La primerarepresenta la observación y la teo ría y la se gun da la experiencia y la aplicación.

Aunque estos son calificativos muy comunes de la investigación, para al gu nos cien tí fi cos no han cien cia pura o cien cia apli-

1 Investigadora del Centro Universitario de Investigaciones Bibliotecológicas cada, toda es apli ca da y se es ta ble ce una dis tin ción se gún el uso que se dé a los re sul ta dos de una in ves ti ga ción; si se usan para generar nuevos conocimientos se habla de usos internos de la investigación, si se utilizan para aplicación directa, de usos externos. Los resultados de uso interno los utiliza el propio investigador, en tanto que los de uso externo salen de su ámbito.

La investigación no sólo es una actividad antigua del hombre, sino que con ti núa ha cién do se y ad qui rien do ma yor im por tan cia para el desarrollo socioeconómico de los países.

En la épo ca mo der na, los paí ses de los si glos XVII y XVIII, propor cio na ron por vez pri me ra las ba ses de su rea li za ción, pri me ro como pro tec to res el nue vo sa ber y más tar de con la es pe ran za de que se produjeran descubrimientos e invenciones de valor mi litar, industrial o mé di co. En la épo ca ac tual, la co rrien te de in novaciones del conocimiento, de productos y de procesos ha llevado a crear organismos especializados nacionales e internacionales de investigación tanto oficiales como privados.

La investigación científica y humanística es muy costosa para que un investigador pueda financiarla individualmente, por eso los trabajos de penden de sub ven ciones otorgadas, ge neralmente, por organismos gubernamentales.

"La re la ción en tre la teo ría y la prác ti ca que ca rac te ri za a la universidadcontemporánea es una herenciamedieval que adquiere su pleni tud en el Renaci mien to, se im pone a la en se ñan za uni versi ta ria la obli ga ción de no li mi tar se a la con ser va ción de lo an ti- 
guo, sino de ex plo rar nue vos te rre nos, profun di zary ex ten der las investigaciones, hacer progresar a la ciencia”. (Asti Vera)

En Mé xi co, como en mu chos otros paí ses, la in ves ti ga ción se ha realizado tra dicional men te en las uni versidades y en or ga nis mos gubernamentales especializados, y no a través de institutos o academias independientes. La misión de elaborar y ejecutar la política cien tífica ha es ta do a car go de ins ti tu tos na cio na les, actualmente del Consejo Nacional de Ciencia y Tecnología (CONACYT), que debe bus car una políti ca cohe ren te y equi li brada para la asignación de fondos que apoyen a la investigación.

En 1988 se estimaba que entre el 60 y el $90 \%$ de las actividades cien tíficas en diferen tes áreas selle va ba a caboen la Uni ver si dad Nacional Au tó no ma de Mé xi co (UNAM). En 1987 ocu pó el segun do lu gar en la asig na ción de re cur sos para in ves ti ga ción, entre las 10 mayoresinstituciones que ejercen estos recursos, sólo fue superada por el Instituto Mexicano del Petróleo.(Villa)

La Ley Orgánica de la UNAM en su artículo primero establece como una de sus finalidades básicas “organizar y realizarinvesti gaciones, en es pe cial acerca de las con di cio nes y proble mas nacionales". Estas funciones son realizadas principalmente en los 35 ins ti tu tos y cen tros de in ves ti ga ción con que cuen ta: 14 ins titu tos y sie te cen tros en elárea cien tífica, nue ve ins ti tu tos y cin co cen tros en elárea hu ma nís tica. En 1988 el gas to en in ves ti ga ción representó el $21.87 \%$ del presupuesto total de la Universidad. Según declaraciones de la Secretaría de Educación Pública (SEP), pu bli ca das el 22 de ene ro de 1990, el 67\% de las in ves tiga cio nes del país se rea li zan en la UNAM, el $10 \%$ en la UAM y el $23 \%$ en las universidades de los estados. (Llinas Zárate)

La UNAM cuenta con 2,652 investigadores, de los cuales 855 son del sub sis te ma de in ves ti ga ción hu ma nís ti ca, pero, sólo 471 son de tiempo completo; 1,797 pertenecen al subsistema de inves ti ga ción cien tífi ca con 840 de tiem pocom ple to. La SEP se ñala que uno de los obstáculos, que sólo paulatinamente se ha superado y que afecta al desarrollo de la investigación y de los estudios de posgrado, es la escasa vinculación entre las instituciones educativas y el sector productivo o práctico.

En las conferencias te má ti cas, ce le bra das en la UNAM con motivo del Congreso Universitario, especialistas que se ocuparon de la in ves ti ga ción seña la ron una se rie de proble mas que afec tan el de sa rro llo de las ta reas de in ves ti ga ción en la Uni ver si dad. Entre otros, mencionaron el reducido número de investigadores el raquítico apoyo que el Estado dedica a esta actividad $(0.4 \%$ del PIB), el deterioro impresionante de los salarios, las numerosas ba rre ras que han que ven cer para ha cer una ca rre ra de in ves ti gador, y el aislamiento de la investigación, resaltando su separación de la docencia, y el trabajo individualista de los investigadores que estimula la formación de personajes de las ciencias y no la de cuadros de investigadores, aun cuando está pro ba do que el tra bajo co lec ti vo es mu cho más pro duc ti vo que el trabajo individual.
¿Quién uti li za los re sul ta dos de la in ves ti ga ción que se reali zaen la Universidad? Mario Bunge dice que cuando algún investigador ha re suel to un pro ble ma, lo más pro ba ble es que el pro pio investigador u otra persona, inmediatamente o poco después, sus ci te toda una nue va se rie de cues tio nes proble máti cas re la ciona das con los re sul ta dos. La in ves ti ga ción es una reac ción en cadena au to sos te ni da acau sa del carác terimperfec toy sis te mático del conocimiento.

La mar cha de la cien cia con sis te en cons truir nue vos proble mas sistemáticos, más que en descubrir problemas existentes, estos nuevos problemas suelen ser de mayor profundidad. El mejor criterio para averiguar si una disciplina sigue viva consiste en mirar si aun plantea problemas nuevos, problemas cuyas soluciones aumentarían sustancialmente nuestro conocimiento. Así ve mos que uno de los usua rios de los re sul ta dos de las in ves ti gaciones es el propio investigador.

El resultado de los estudios del subsistema de la investigación humanística, aun que se ha men cio na do el poco con tac to de ésta con la do cen cia, es usa do para apo yar la do cen cia y la in ves ti gación. Tam bién hay in ves ti ga ción para uti li zar se en a pla nea ción y apli ca ción de so lu cio nes a los pro ble mas na cio na les, como serían los trabajos que realizan los Institutos de Investigaciones Sociales, Jurídicas, Históricas, Estéticas, etc. Por ejemplo el banco de información sobre legislación (UNAM-JURE), resultado de una investigación biblio gráfica, es de gran uti li dad para numerosas disciplinas.

Tal vez no de los mo ti vos por el cual los re sul ta dos de la in ves tigación son poco utilizados por usuarios no universitarios es la fal ta de una bue na di fu sión y ade cuaos ca na les de co mu ni ca ción. Ade más to da vía per sis te en gran me di da la cos tum bre de ig no rar a la in ves ti ga ción mexi ca na, au na da al poco apre cio que el in vesti ga dor mexi ca no tie ne por la in for ma ción ge ne ra da por sus colegas nacionales. Sería interesante saber la frecuencia con que nues tros in ves tiga dores in clu yen en sus lec tu ras lo pu blica do por otros in ves ti ga dores mexi ca nos, el grado de cre dibilidadqueles conceden y las veces en que esa información ha contribuido a que el investigador genere nueva información.

Los me dios para dar a co no cer los re sul ta dos de la in ves ti ga ción son variados; los tradicionales son los reportes internos, los trabajosenrevis tas es pe cializadas, con gre sos, simposiay en libros. Éstos unen a los investigadores entre ellos. Pero no necesariamen te con aque llos que es tán en po si bi li da des de po ner en práctica los resultados de la investigación. Este medio tradicional también presenta el problema tiempo que media entre la redacción de un ar tícu lo y su pu bli ca ción en una re vis ta o en un libro.

Un me dio in for mal, pero más in me dia to y de gran im pac to, es el contacto personal en conferencias, reuniones y seminarios, y a través de comunicaciones directas entre los investigadores por medio de correspondencia, que ahora con el correo electrónico, fax y te les es mu cho más efec ti va; el uso del te lé fo no es tam bién pri mor dial para la co mu ni ca ción de lo que se co no ce como "cole gios in visibles". Elincremen to de las conferencias, se mi narios y re u nio nes ha sido es pec ta cu lary sibien son tras cen den ta les por 
los tó pi cos que se ex po nen en ellos, lo son más por los con tac tos personales.

Los adelantos tecnológicos han proporcionado nuevos medios para al ma ce nar, ac ce der y di se mi nar los re sul ta dos de la in ves tigación. Los investigadores intercambian información por computadora, usaninformaciónal macenadaenCD-ROM, consultan los bancos de datos en línea, etc.

Pero ¿cómo se difunde la investigación que se realiza en la UNAM? Se dice que las pu bli ca cio nes por más rá pi das que sean sólo dan al investigador una imagen del pasado, es esencial por tan to te ner in for ma ción al día acer ca de los tra bajos de in ves ti gación que se es tán efec tuan do. A este res pec to en los paí ses avanzados se publican semanalmente, newsletters o research progress que mantienen a los investigadores informados sobre los avan ces en de ter mi na da dis ci pli na. La UNAM publi ca y tiene en línea un ban co de da dos so bre las in ves ti ga cio nes en proceso llamado ARIES, lamentablemente es poco confiable y la información que proporciona no está actualizada. También en los re por tes anua les de la UNAM apa re cen esos da tos, así como en ca tálo gos que publican los sub sis te mas de in ves ti ga ción científica y humanística.

En cuan to a la di fu sión de las in ves ti ga cio nes ya ter mi na das, éstas se hacen por las vías tradicionales de revistas, libros, informes técnicos, memorias de congresos, etc. El Centro de In for ma ción Cien tífica y Hu ma nís ti ca por su par te tie ne unabase de da tos que re gis tra las in ves ti ga cio nes que se lle van a cabo en la UNAM. Todo esto, sin em bar go, no es su fi cien te, la in for mación es ca se ra, atra sa da y no hay me ca nis mos efec ti vos para dar a conocer los resultados de la investigación universitaria a otras instituciones del país y del extranjero.

Las carac te rís ti cas de las re vis tas na cio na les son, por otra par te: poca difusión,tirajes cortos, apa re cen con re tar do y a ve ces son suspendidas por largas temporadas. Esto se debe al poco apoyo que existe por parte de las instituciones para publicarlas.

Con re la ción a la in ves ti ga ción bi blio te co ló gi ca en nues tro país, es una actividad joven que a pe sar de su cor ta tra yec to ria ha experimen ta do uncre cimien to progre si vo y ha di versifica do su temática. El Centro Universitario de Investigaciones Bibliotecológicas, fundado en 1981, se ha propuesto plantear problemas nuevos relacionados con el desarrollo de la bibliotecología mexicana para generar conocimientos que mantengan vivoelin terés en nues tra disciplina. Realizain ves ti gación dirigi $\mathrm{da}$ a re sol ver los pro ble mas que en fren ta el ejer ci cio de la pro fesión en el país. Por tan to, la in ves ti ga ción que ha he cho el CUIB es esencialmente aplicada y utilizada también como apoyo a la en se ñan za La di fu sión de la in ves ti ga ción rea li za da en elCUIB se hace por me dio de la re vis ta InvestigaciónBibliotecológica, y a través de las publicaciones de cuadernos y libros. Se hace publicidad de ellos en los órga nos de la es pecialidad. La men tablemente la revista adolece de las mismas fallas que presentan las revistasmexicanas, aparece con retraso y su difusión es muy limitada.

Aunque las investigaciones en proceso se difunden a través de los me dios de la UNAM ya se ña la dos, y en la pu bli ca ción in gle sa Cu rrent Re search in Li brary and In for ma tion Science, se ría útil para la co mu ni dad bi blio te ca ria dar las a co no cer a tra vés del $\mathrm{No}$ ticiero de AMBAC, del Boletín Informaciones del Colegio Nacional de Bibliotecarios y en la propia revista del CUIB.

En un es tu dio rea li za do en In gla te rra so bre la di fu sión de los resul ta dos de lain ves ti ga ción en la profe sión biblio te caria, selle gó a re sul ta dos muy in tere san tes. Porejem plo, las publicaciones periódicas superaron a los contactos personales como medio para mantenerse informados; los encuestados consideraron que una publica ciónimpi de la am bi güe dad y las ma las in ter pre ta ciones y tiene una circulación más am plia. Las re vis tas fue ron el sis te ma más uti li za do por los que bus can in for ma ción y por los que la diseminan. Publicaciones como newsletters, también resultaron populares como material de lectura, representaron una herramienta muy útil para difundir la investigación en proceso y los reportes finales, te nien do más im pac to como ele men to de aler ta que las publicaciones secundarias; índices y abstracts.

Si en Mé xi co se hi cie ra una en cues ta pa re ci da, los re sul ta dos serían muy diferen tes por la es ca sez de publi ca cio nes pe rió di cas de la es pe cia li dad, por que no exis ten ín di ces que ana li cen lo pu blica do en con gre sos, se mi na rios y re vis tas y por que ca re ce mos de alguna publicación parecida a los newsletters.

En conclusión, podemos decir que se necesita a nivel nacional, más y mejor difusión de la investigación que se realiza en la UNAM para man te nerin for ma dos a los in ves tiga do res del país y de esta ma ne ra, im pe dir du pli ci dad en las in ves ti ga cio nes y para que ésta se co noz ca en los me dios uni ver si tarios. A nivelin ternacional es muy importantehacer conocer la in vestigación que se rea li za en el país por que la bi blio te co lo gía es una cien cia que no tie ne fron te ras, y ade más pue de con tri buir a su de sa rro llo de manera más eficiente.

\section{OBRAS CONSULTADAS}

1. Asti Vera, Armando. Metodología de la investigación. Buenos Aires, Kapelusz, 1973. 191p.

2. Bunge, Mario. La investigación científica. Barcelona, Ariel, 1973. p. 230-239. 
3. Córdova, Arnaldo. "La investigación en la Universidad" Cuadernos del Congreso Universitario. 23 de enero de 1990. p. 23 -31. (Conferencias temáticas, 16)

4.Fergu son, Dou glas. "Difu sión de las in ves ti ga ciones de biblio te co lo gía y cien cia s de la in for ma ción de los Es ta dos Uni dos de amé rica” Bol. UNESCO Bibl. v. 29, no. 6, nov-dic, 1975. p. 339-349.

5. Information re search, re searchmethods in libraryand information science. Ed. By Neva Tu dor-Si lo vic and Ivan Mihel. Lon don, Taylor Graham, 1988. 261 p.

6. Kllinas Zárate, Isabel. En “UNAM 67\% de las investigaciones...” Uno más Uno. 22 de enero 1990. p. 3

7. Lynam, Peter, et. Al. Research and the practitioner. Dissemination of research results within the library information profession. London, ASLIB, 1982, 83p. (ASLIB Occasional Publication, 27)

8. Peimbert, Manuel. "Investigación e identidad nacional". Cuadernos del Congreso Universitario. 23 de enero de 1990. p. $32-36$ (Conferencias Temáticas, 16)

9. Samaha,Emile. "Lain for ma ción sobrelas in vesti ga cionesen cur so de realización”. Bol. UNESCO Bibl.v. 32, no. 5, sep t- oct 1978. p. 347-357.

10. Vil la-s,m Juan Car los, et al. "Gas to y po lí ti cas de in ves ti ga ción en la UNAM”. CienciayDe sarrollo. Año XIV, no. 80, ma yo- jun 1988. p. 93-112.

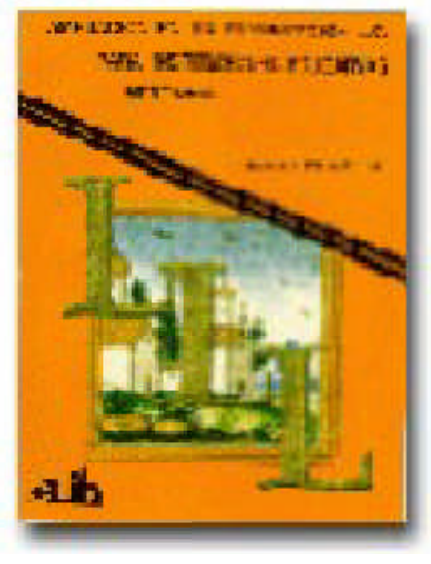

Ampliación de la Clasificación L. C. para instituciones educativasmexicanas. Martínez Filiberto. 50 p. 